\title{
Single-wavelength anomalous dispersion phasing for Serial Millisecond Snapshot Crystallography
}

\author{
S. Botha ${ }^{a, b, *}$, J. Martin-Garciaa,c, H. Hu ${ }^{b, c}$, U. Weierstall b,c, M. Fuchs ${ }^{d}$, W. Shid ${ }^{d}$, \\ B. Andid ${ }^{d}$ J. Skinner ${ }^{d}$, H. Bernstein ${ }^{e}$, P. Fromme ${ }^{b, c}$, N. Zatsepin ${ }^{a, b, *}$ \\ a Department of physics, Arizona State University, Tempe, AZ 85287, USA \\ ${ }^{b}$ Biodesign Center for Applied Structural Discovery, Arizona State University, Tempe, AZ 85287, USA \\ c School of Molecular Sciences, Arizona State University, Tempe, AZ 85287, USA \\ ${ }^{d}$ Energy \& Photon Sciences Directorate, Brookhaven National Laboratory, Upton, NY 11973, USA \\ e School of Chemistry and Materials Science, Rochester Institute of Technology, Rochester, NY, USA \\ * Author contacts: sbotha@asu.edu and nzatsepi@asu.edu
}

Serial femtosecond crystallography (SFX) is an up and coming method for room-temperature protein structure determination [1] and it has been shown that SFX data can be phased de novo [2]. This method of data collection has been adapted for synchrotron sources, termed serial millisecond crystallography (SMX) $[3,4,5]$. SMX substantially reduces radiation damage incurred by the individual protein crystals compared to conventional, oscillation data collection approaches, facilitating room-temperature structure solution from micrometer sized crystals at synchrotrons. However, de novo phase retrieval remains difficult and is rarely applied to serially-collected SFX and SMX data. Here, we present a systematic study of single-wavelength anomalous dispersion (SAD) for de novo phasing of SMX data. This study is aimed at determining optimal experimental parameters for data collection (e.g. energy, exposure, number of patterns), as well as establishing data metrics for successful SAD phasing of serial millisecond snapshot crystallography data.

Using the model system proteinase $\mathrm{K}$, various SMX datasets were collected at the Frontier Microfocusing Macromolecular Crystallography beamline of NSLS-II at the Brookhaven National Laboratory. The anomalous signal strength was investigated along with the SAD phasing potential of the individual datasets. The protein crystals were injected into the X-ray interaction region in a free-standing lipidic cubic phase (LCP) jet for serial snapshot data collection. For native sulphur$\mathrm{SAD}$ phasing, various exposure times and $\mathrm{X}$-ray wavelengths ranging from $7.11 \mathrm{keV}$ down to $5 \mathrm{keV}$ were used. Furthermore, other anomalous scatterers, such as mercury, were also introduced into the crystals and datasets collected below, at and above the absorption edge.

\author{
Acknowledgements \\ SC0012704(KC0401040)). We would further like to acknowledge Stu Myers and Jean Jakoncic.

\section{References} \\ [1] Chapman $\mathrm{H}$, et al. Nature 2011 Feb 3;470(7332):73-7 \\ [2] Barends T. R. M, et al. Nature 2014 Jan 09; 505; 244-247 \\ [3] Gati et al., 2014 IUCrJ 1:87 \\ [4] Stellato et al., IUCrJ. 2014 Jul 1; 1(Pt 4): 204-212, \\ [5] Botha et al., Acta Crystallogr D Biol Crystallogr. 2015 Feb;71(Pt 2):387-97
}

This research was supported by NSF award \#1565180 and BioXFEL STC Award \#1231306. Work at the AMX (17-ID-1) and FMX (17-ID-2) beamlines is supported by the NIH National Institute of General Medical Sciences (P41GM111244), and by the DOE Office of Biological and Environmental Research (KP1605010), and the NSLS-II at BNL is supported by the DOE Office of Basic Energy Sciences (DE- 